\title{
Why Do They Fall? The Impact of Insomnia on Gait of Older Adults: A Case-Control Study
}

This article was published in the following Dove Press journal: Nature and Science of Sleep

\section{Dani Kirshner' \\ Rachel Kizony ${ }^{2,3}$ \\ Efrat Gil \\ Kfir Asraf ${ }^{4}$ \\ Tal Krasovsky $\mathbb{D D}^{5,6}$ \\ Iris Haimov ${ }^{7}$ \\ Tamar Shochat ${ }^{8, *}$ \\ Maayan Agmon ${ }^{8 *}$}

'Clalit Health Services; Faculty of Medicine, Technion, Haifa, Israel; ${ }^{2}$ Occupational Therapy Department, University of Haifa, Haifa, Israel;

${ }^{3}$ Occupational Therapy Department, Sheba Medical Center, Tel- Hashomer, Tel-Aviv, Israel; ${ }^{4}$ Department of Psychology, University of Haifa, Haifa, Israel; ${ }^{5}$ Physical Therapy Department, University of Haifa, Haifa, Israel;

${ }^{6}$ Pediatric Rehabilitation Department, Sheba Medical Center, Tel- Hashomer, Tel-Aviv, Israel; ${ }^{7}$ The Center for Psychobiological Research, Department of Psychology, The Max Stern Yezreel Valley College, Yezreel Valley, Israel;

${ }^{8} \mathrm{School}$ of Nursing, Faculty of Health and Social Welfare, University of Haifa, Haifa, Israel

*These authors contributed equally to this work

Correspondence: Maayan Agmon Email magmon@univ.haifa.ac.il
Study Objectives: To compare gait and cognitive performance conducted separately as a single- (ST) and simultaneously as a dual-task (DT), ie, when a cognitive task was added, among community-dwelling older adults with and without insomnia.

Methods: Participants included: 39 (28 females) community-dwelling older adults with insomnia, 34 (21 females) controls without insomnia. Subject groups were matched for age, gender, and education. Sleep quality was evaluated based on two-week actigraphy. Gait speed and cognition were assessed as ST and DT performance. DT costs (DTCs) were calculated for both tasks. Outcomes were compared via independent samples $t$-tests or Mann-Whitney $U$-tests.

Results: Older adults with insomnia demonstrated significantly slower gait speed during ST $(1 \pm 0.29$ vs $1.27 \pm 0.17 \mathrm{~m} / \mathrm{s}, p<0.001)$ and DT $(0.77 \pm 0.26$ vs $1.14 \pm 0.20 \mathrm{~m} / \mathrm{s}, p<0.001)$ and fewer correct responses in the cognitive task during ST ( $21 \pm 7$ vs $27 \pm 11, p=0.009)$ and DT ( $19 \pm 7$ vs $23 \pm 9, p=0.015$ ) compared to control group. DTC for the gait task was higher among older adults with insomnia (18.32\%, IQR: 9.48-30.93 vs 7.81\% IQR: 4.43-14.82, $\mathrm{p}<0.001)$. However, no significant difference was observed in DTC for the cognitive task (14.71\%, IQR: $-0.89-38.84$ vs $15 \%$, IQR: $-0.89-38.84 \%$, p=0.599).

Conclusion: Older adults with insomnia have lower gait speed and poorer cognitive performance during ST and DT and an inefficient pattern of task prioritization during walking, compared to counterparts without insomnia. These findings may explain the higher risk of falls among older adults with insomnia. Geriatric professionals should be aware of potential interrelationships between sleep and gait.

Keywords: actigraphy, sleep quality, gait, dual-task, DT

\section{Introduction}

Insomnia, a disorder in initiating and maintaining sleep and impaired daytime functioning, has recently received much attention. ${ }^{1,2}$ It has an annual incidence rate of $36 \%,{ }^{3}$ with one-third of the population reporting at least one insomnia symptom. ${ }^{4}$ The prevalence of insomnia increases with age, ${ }^{5}$ and it is the most common sleep complaint in older adults., ${ }^{4,6}$ Adults with insomnia commonly experience impaired attention, ${ }^{7}$ memory, ${ }^{8}$ mood, ${ }^{9}$ and postural control, ${ }^{10}$ and as much as a 4.5 -fold higher risk of falls. ${ }^{11-13}$

Due to this alarmingly high risk of falls among people with insomnia, a few lines of studies have been conducted aiming to understand the association between sleep, falls, postural control and gait. Studies that have investigated this link can generally be divided into four main categories based on their research questions and methodologies: (1) Population-based studies that demonstrate the link between falls and sleep 
disturbances. ${ }^{10,14}$ These studies were pioneering in illuminating this link, yet causality could not be inferred due to their reliance on self-report of both sleep quality and falls and due to their cross-sectional design. (2) Studies assessing the effects of laboratory-based sleep deprivation protocols on posture among healthy and typically younger adults. ${ }^{15,16}$ Such protocols, demonstrating the negative effects of sleep deprivation on postural control, have low ecological validity as they ignore the wide-ranging nature of chronic sleep disturbance and its continuous effects on postural control. (3) Studies exploring the link between posture and sleep among specific clinical populations, for example, among people with obstructive sleep apnea ${ }^{17}$ or Parkinson's disease. ${ }^{18}$ These studies have advanced the science by not only demonstrating the mutual relationships between sleep and postural control (mainly gait quality) but also reveal potential mechanisms underlying the relationships between these domains; however, generalizability is limited due to the unique types of morbidities characterizing the samples. (4) Studies that objectively evaluate both sleep and postural control (operationalized as gait) among healthy subjects in a cross-sectional design ${ }^{19,20}$ Although the mechanisms are yet to be revealed, these latter studies pave the way to the investigation of shared mechanisms underlying the link between sleep and postural control, specifically gait.

Gait involves a delicate equilibrium between motor and cognitive control, specifically executive function. ${ }^{21}$ The most common paradigm to examine the central control of mobility, ie, the relationships between the motor and cognitive aspects of gait, is the Dual-Task (DT) paradigm. In recent years, growing evidence has shown that performance during DT gait serves as a behavioral marker for Parkinson's disease, Alzheimer's disease, ${ }^{22}$ accelerated aging, ${ }^{23}$ and falls. ${ }^{24-26}$ This paradigm requires an individual to perform a primary task (eg walking) while carrying out a concurrent secondary (eg cognitive) task. Of interest is the change in a given variable (eg gait speed, cognitive performance) between when the task is performed alone, namely, as a single task (ST), and when it is performed simultaneously with another task (dual task - DT). The difference between ST and DT performance is quantified as "DT cost" (DTC), also termed the DT effect, ${ }^{27,28}$ reflecting a higher demand for cognitive and specifically executive control resources. ${ }^{21}$ DTC can thus explain the manner in which one divides attentional resources between the motor and cognitive aspects of the task.

The DT paradigm has been used to explore gait mechanisms, especially during the aging process, ${ }^{29}$ and to assess prioritization strategies between motor and cognitive demands that are required to cope with real-life DT gait situations such as walking while talking. ${ }^{30}$ DT paradigm studies have shown that, unlike younger adults, older adults sometimes tend to adopt a "cognitive first" prioritization strategy, at the cost of reduced motor control. ${ }^{31}$ This strategy may be particularly taxing in older adults with insomnia, given the negative impact of insomnia on cognitive performance and specifically on attention. ${ }^{8}$

Considering the higher prevalence of falls among older adults with insomnia, ${ }^{10-14}$ a modifiable sleep disorder, and the importance of DT gait as a marker for age-related adverse outcomes including falls, ${ }^{24-26}$ a comprehensive comparison of gait characteristics under ST and DT between older adults with and without insomnia is vital. Thus, the aim of this study was to compare gait and cognitive performance under single and dual-task conditions among community-dwelling older adults with and without insomnia. We hypothesize that cognitive and motor (gait) performance, both as single and as dual tasks, would be inferior in older adults with insomnia compared to their no-insomnia counterparts. Furthermore, we hypothesize that the dual-task cost will be higher in the insomnia group in both the cognitive and motor domains.

\section{Methods}

\section{Participants and Procedures}

This study compared two samples of high-functioning, community-dwelling older adults with similar demographic background and from the same geographical area. In both samples, participants completed a screening questionnaire to establish eligibility, and the Pittsburgh Sleep Quality Index (PSQI), wore an activity monitor (actigraph) for objective sleep measurements, and underwent a gait and cognitive task as an ST and DT using standard protocols (see below). The study sample was comprised of participants screened positive for insomnia based on standard DSM-5 criteria ("insomnia group"), and the control sample was comprised of participants with no known sleep disturbances or complaints based on self-report ("no-insomnia group").

Exclusion criteria for both groups at the time of enrollment were assessed based on self-report and included: (1) the presence of a neurologic diagnosis, such as cerebral vascular accident, Parkinson's disease, Alzheimer's disease, or multiple sclerosis; (2) severe orthopedic restrictions such as acute back pain, recent fractures, or a total hip replacement; and (3) significant hearing or vision loss. 
Additional exclusion criteria in the insomnia group included the presence of obstructive sleep apnea or periodic limb movements in sleep.

The study was approved by the ethics committee of the Faculty of Social Welfare and Health Sciences at the University of Haifa. Approval \# 20/320. All participants signed an informed consent.

\section{Insomnia Group}

39 older adults (11 males, 28 females) aged 65 and above were recruited for a study calling for older adults with insomnia. Eligibility was based on an initial screening questionnaire and on 2 weeks of objective sleep measurement by actigraphy. Volunteers were eligible if: (1) they reported sleep dissatisfaction characterized by difficulty initiating and maintaining sleep at least 30 minutes per night on at least 3 nights per week, and that this condition lasted at least several months; and (2) at least one of three commonly used benchmark criteria for insomnia was met on at least 3 nights per week based on actigraphy: sleep onset latency $(\mathrm{SL})>30$ minutes, wake after sleep onset (WASO) $>30$ minutes, or sleep efficiency (SE) $<85 \%$. ${ }^{32,33}$

\section{No-Insomnia (Control) Group}

34 participants (13 male, 21 female) originally recruited for a previous observational study ${ }^{20}$ with similar methodology and similar eligibility criteria in terms of demographics, health, and cognitive and motor functional status (with the exception of sleep status) served as controls. Participants reported that they were free of any sleep disturbances. This was corroborated by one week of actigraphy monitoring, showing sleep measures indicating overall good sleep quality.

Demographic comparisons confirmed that the groups did not differ by age, years of education, and gender (see Table 1).

\section{Sleep Assessments}

Sleep quality was assessed by the Pittsburgh Sleep Quality Index (PSQI), ${ }^{34}$ a widely used self-rated questionnaire that assesses sleep quality over the past month, validated for Hebrew speakers. ${ }^{35}$ Seven component scores are computed on a scale between 0-3 to assess subjective sleep quality, sleep latency, sleep duration, habitual sleep efficiency, sleep disturbances, use of sleeping medication, and daytime dysfunction. The sum of scores results in the global PSQI score, ranging from 0 to 21 , with higher scores representing poorer sleep, and a cutoff score $>5$ defined as poor sleep quality.

Objective assessment of sleep was obtained by wristworn activity monitors during the night for two weeks in the insomnia group and for one week in the no-insomnia group. As the groups were amalgamated from two separate investigations, activity monitors were not identical. Thus, ActiWatch Spectrum Pro devices (Phillips Respironics, Actiware software version 6.0.9) were used for the insomnia group, and Motionlogger Actigraph devices (Ambulatory Monitoring, Inc., ActionW software, version 2, UCSD algorithm) were used in the no-insomnia group. Sleep measures obtained from both software algorithms using 1-minute epochs included sleep efficiency (SE, percentage of total sleep time after initial sleep onset), sleep onset latency (SL, minutes from lights out to sleep onset) wake after sleep onset (WASO, minutes of wake after sleep onset), and sleep duration (duration of the sleep interval). To determine sleep onset and offset times, participants in both groups completed a sleep log, and those in the insomnia group were also requested to press an event marker button. The accuracy of actigraphy devices is $90 \%$ compared to polysomnographic (PSG) recordings. ${ }^{36-38}$ Additionally, sleep medication use was assessed by selfreport.

\section{Gait Assessment Under Single-Task (ST) and Dual-Task (DT) Performance}

The Mobility Lab System APDM (http://www.apdm.com) was used to measure gait speed. Participants wore three APDM OPAL wireless sensors while walking forward. Participants were instructed to walk on their comfortable speed for 1 minute on a flat surface. Each task, ie, the walking and the cognitive tasks, was performed twice: once as an ST and once as a DT. For the cognitive task, participants were required to subtract repeatedly by 3 from a random number between 100 and 250. In addition, participants were asked to perform the cognitive subtraction task while sitting in a chair as an ST and to speak their answers out load. Tasks were administered in a random order and the number of correct responses was calculated. The subtraction-by-3 task is an internal mental processing task that has been shown to generate higher cognitive load and affect gait performance more negatively than other tasks. ${ }^{28}$ The subtraction-by-3 task is considered to be a sensitive measure and is commonly used in DT walking studies, enabling standardization and comparison between studies. ${ }^{28}$

\section{Dual-Task Cost (DTC) Assessment}

DTC is the relative change in performance between single and dual-task and is calculated using the formula: [(ST$\mathrm{DT}) / \mathrm{ST}] * 100$. 
Table I Demographic and Sleep Characteristics

\begin{tabular}{|c|c|c|c|c|c|}
\hline Demographic Characteristics & No-Insomnia $(n=34)$ & Insomnia $(n=39)$ & $t / \chi 2(d f)$ & $p$ & Effect Size \\
\hline Age (years) & $\begin{array}{l}M=72 \\
S D=6\end{array}$ & $\begin{array}{l}M=74 \\
S D=6\end{array}$ & I.65 (7I) & 0.101 & - \\
\hline Years of education & $\begin{array}{l}M=14 \\
S D=3\end{array}$ & $\begin{array}{l}M=16 \\
S D=3\end{array}$ & $1.79(65)$ & 0.076 & - \\
\hline$\%$ of females & $62(n=21)$ & $72(n=28)$ & $0.82(1)$ & 0.362 & - \\
\hline$\%$ use of sleep medicine & $12(n=4)$ & $38(n=14)$ & $6.36(1)$ & 0.011 & 0.299 \\
\hline Sleep Measures & & & $\cup(Z)$ & $p$ & Effect size \\
\hline Sleep Efficiency (\%) & $\begin{array}{c}\text { Med }=93 \\
I Q R=6\end{array}$ & $\begin{array}{c}\text { Med }=79 \\
\mathrm{IQR}=9\end{array}$ & $114(-6.07)$ & $<0.001$ & 0.505 \\
\hline Sleep Latency (min) & $\begin{array}{l}\text { Med }=9 \\
\mathrm{IQR}=7\end{array}$ & $\begin{array}{l}\text { Med }=17 \\
I Q R=17\end{array}$ & $315(-3.84)$ & $<0.001$ & 0.203 \\
\hline WASO (min) & $\begin{array}{l}\text { Med }=15 \\
I Q R=17\end{array}$ & $\begin{array}{l}M e d=45 \\
I Q R=31\end{array}$ & $173(-5.4 \mid)$ & $<0.001$ & 0.402 \\
\hline Sleep Duration (min) & $\begin{array}{c}M e d=404 \\
I Q R=76\end{array}$ & $\begin{array}{c}M e d=408 \\
I Q R=85\end{array}$ & $631(-0.35)$ & 0.723 & - \\
\hline
\end{tabular}

Notes: A comparison of demographic and sleep characteristics between groups. Effect size are phi ( $\varphi$ ) for Pearson's chi-squared tests and eta-squared $\left(\eta^{2}\right)$ for MannWhitney U-tests.

Abbreviations: WASO, wake after sleep onset; M, mean; SD, standard deviation; Med, Median; IQR, interquartile range.

\section{Statistical Analysis}

Demographic data including age, years of education, and gender as well as sleep measures were compared between groups via independent samples $t$-tests and Pearson's chisquared tests. Outcome measures included ST/DT gait speed, ST/DT number of correct responses on the cognitive task, and dual-task costs (DTC) of both gait speed and correct cognitive responses. Outcome measures were compared via independent samples $t$-tests or Mann-Whitney $U$-tests. Estimators of effect size were Hedges' $\mathrm{g}$ for independent samples $t$-tests, eta-squared $\left(\eta^{2}\right)$ for Mann-Whitney $U$-tests, and phi $(\varphi)$ for Pearson's chi-squared tests. Effect sizes were computed only for significant comparisons.

\section{Results}

Group comparisons of the demographic variables and actigraphy-derived sleep measures are presented in Table 1. No group differences were found for age, years of education, or gender, but the insomnia group had a higher prevalence of sleep medication use. The analysis of the sleep measures revealed, as expected, that the insomnia group had significantly higher SL and WASO and significantly lower SE, compared to the no-insomnia group. No differences were found for sleep duration. All significant comparisons had a large effect size.
Analysis of the outcome measures is presented in Table 2 and is demonstrated in Figure 1A-C. Overall, the insomnia group demonstrated significantly slower gait speed in ST and in DT and had a higher DTC for gait speed compared to the no-insomnia group, respectively. The insomnia group also had a significantly lower number of correct responses in the cognitive task during ST than the no-insomnia group. However, the insomnia group was not found to be different from the no-insomnia group in DTC for the cognitive task. Moreover, to eliminate the effect of sleep medication on our findings, we have conducted a sensitivity analysis while excluding 18 participants who used sleep medication (14 from the insomnia group and 4 from the no-insomnia group). Results remained the same, the insomnia group had slower gait speed in both ST $[\mathrm{t}(53)=-5.22, \mathrm{p}<0.001]$ and DT $[\mathrm{t}(53)=-5.19, \mathrm{p}<0.001]$, and had higher DTC for gait speed $[\mathrm{U}=240, \mathrm{Z}=-2.28, \mathrm{p}=0.022]$. The insomnia group had fewer correct responses than the control group at both ST $[\mathrm{t}(53)=$ $-2.42, p=0.018]$ and DT $[t(53)=-2.36, p=0.021]$. Finally, the groups did not differ in the dual-task cost for the cognitive task $[\mathrm{U}=372, \mathrm{Z}=-0.05, \mathrm{p}=0.959]$.

\section{Discussion}

The results of our study demonstrate that communitydwelling older adults with insomnia have significantly slower 
Table 2 A Comparison Between ST and DT Gait and Cognition

\begin{tabular}{|c|c|c|c|c|c|}
\hline Gait Speed (meter/second) & No-Insomnia $(n=34)$ & Insomnia $(n=39)$ & $t(d f) / U(Z)$ & $p$ & Effect Size \\
\hline ST & $M=1.2 S D=0.1$ & $M=I S D=0.2$ & $-4.72(62.83)$ & $<0.001$ & 1.07 \\
\hline DT & $M=I . I S D=0.2$ & $M=0.7 \mathrm{SD}=0.2$ & $-6.64(7 I)$ & $<0.001$ & 1.55 \\
\hline DTC (\%) & $\begin{array}{c}M e d=7 \\
I Q R=10\end{array}$ & $\begin{array}{l}M e d=18 \\
I Q R=2 I\end{array}$ & $321.5(-3.77)$ & $<0.001$ & 0.195 \\
\hline Cognition (Number of correct responses) & & & $t(\mathrm{df}) / \mathrm{U}(\mathrm{Z})$ & $p$ & Effect size \\
\hline ST & $M=27 S D=11$ & $M=21 S D=7$ & $-2.69(55.99)$ & 0.009 & 0.64 \\
\hline DT & $M=23 S D=9$ & $M=19 S D=7$ & $-2.49(7 \mathrm{I})$ & 0.015 & 0.58 \\
\hline DTC(\%) & $\begin{array}{l}M e d=14 \\
I Q R=39\end{array}$ & $\begin{array}{l}M e d=15 \\
I Q R=4 \mid\end{array}$ & $615.5(-0.52)$ & 0.599 & \\
\hline
\end{tabular}

Note: Effect size are hedge's $g$ for $t$-tests and eta-squared for Mann-Whitney U-test.

Abbreviations: ST, single task; DT, dual task; M, mean; SD, standard deviation; Med, median; IQR, interquartile range, DTC; dual task cost.

gait speed and lower cognitive performance during ST and DT, compared to an age-, education-, and gender-matched group of community-dwelling older adults without insomnia. Strikingly, compared to older adults with no insomnia, older adults with insomnia paid a higher price (DTC) in walking speed when under increased cognitive load; however, both groups paid the same price when a motor load (walking) was added to a cognitive task. These findings suggest that older people with insomnia allocate their resources to cognition rather than to gait when challenged with dual tasks, thereby putting themselves at higher risk for falls..$^{39,40}$ To the best of our knowledge, ours is the first study to date that compares gait performance between healthy community-dwelling older adults with and without insomnia while closely scrutinizing divided attention patterns between the tasks.

Our findings are in line with previous studies that also show a negative association between sleep disturbances and gait performance, ${ }^{41,42}$ as well as an association between lower sleep efficiency and decreased gait speed and increased gait variability under dual-task conditions. ${ }^{20}$ Furthermore, several studies have shown that self-reported daytime sleepiness, often a sign of disturbed sleep, was associated with increased pace variability in the DT condition. ${ }^{43-48}$ However, a comprehensive, in-depth assessment regarding the manner in which older adults with insomnia divide their resources between the two domains of gait and cognition (ie, DTC of tasks representing each) is yet to be addressed by most studies. ${ }^{39}$

The lower performance on nearly all task measures observed in this study among participants with insomnia may indicate inadequate attentional resources, ${ }^{39}$ which may lead to a higher risk of falls due to cognitive task prioritization. ${ }^{31,40}$ The model of task prioritization postulates that older adults with lower cognitive reserve capacity, expressed as reduced executive function, may use an inefficient strategy, ie, "cognitive first", rather than the "posture first" strategy, thereby compromising their safety and exposing themselves to an increased risk of falls. ${ }^{40}$ Indeed, reduced executive function, and specifically divided attention, which is strongly required during walking, has been linked to gait abnormalities especially with an added (dual) task. ${ }^{24,25,44,49-52}$ Insomnia and sleep disturbances have also been associated with reduced executive function, ${ }^{53-56}$ that is common in individuals with low cognitive reserve. ${ }^{57}$

Several mechanisms can potentially contribute to reduced executive function and limited attention capacity that may explain why people with insomnia are likely to have a reduced gait performance, leading to a higher risk of falls. These pathways are interconnected and can be divided into three general levels: anatomy, physiology, and emotional status (ie, mood). The interconnection between these three levels can lead to a vicious cycle, as shown in Figure 2. However, implicit causal pathways are yet to be fully described.

Anatomically, several neuroanatomical regions, such as the pontine tegmentum, the pedunculopontine nucleus, and the medial medulla have been offered as potential structures that connect sleep-regulation nuclei and gait-controlling areas. ${ }^{58}$ Additionally, reduced hippocampal volume was linked to poor sleep, ${ }^{59}$ reduced executive function ${ }^{60}$ and reduced gait performance ${ }^{61,62}$ in various studies that explored sleep, executive function or gait disturbances separately. Furthermore, during the aging process, it is typical to observe 

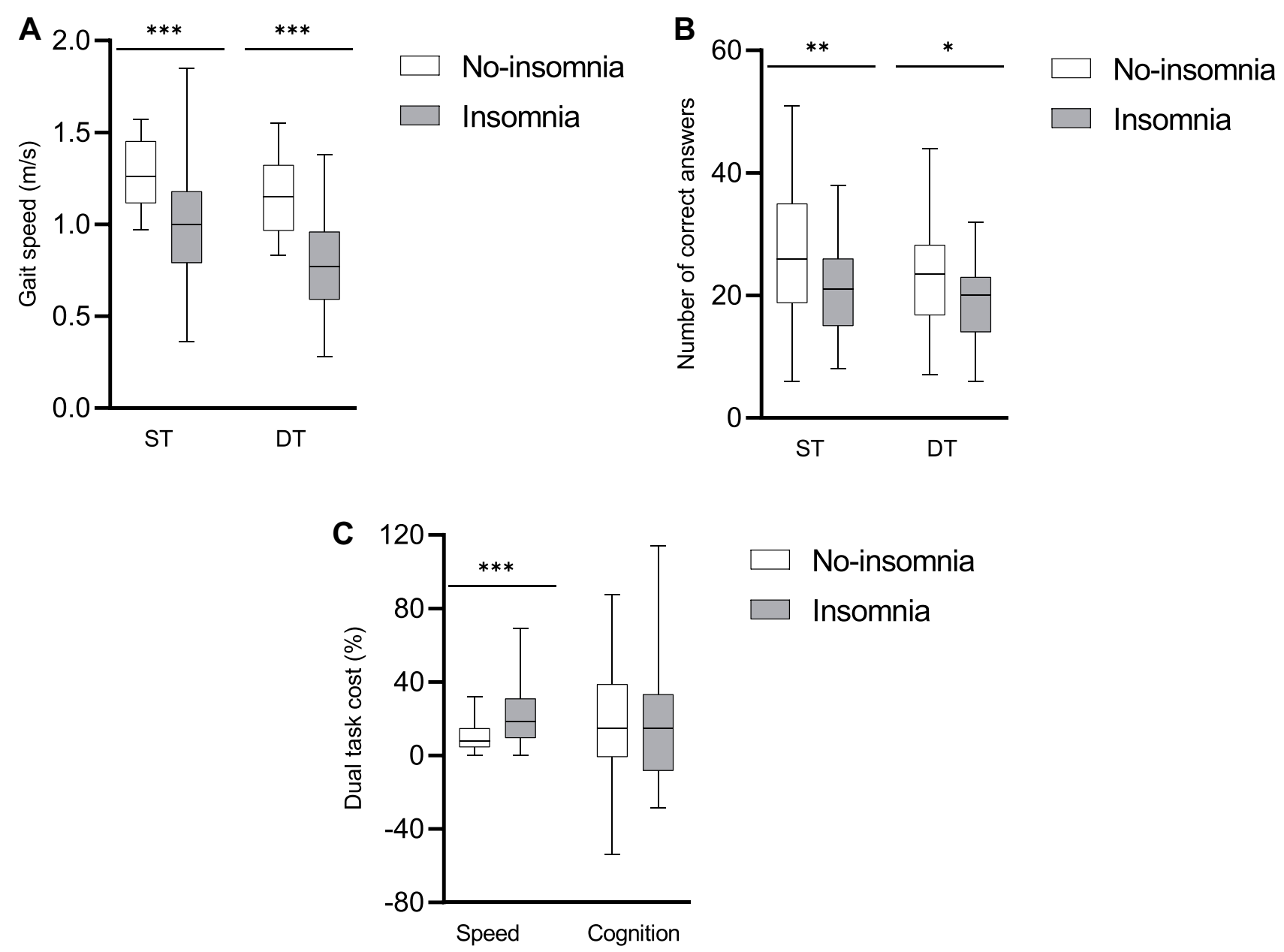

Figure I (A) Comparisons between the insomnia and no-insomnia groups in single-task (ST) and dual-task (DT) gait speed performance. (B) Comparisons between the insomnia and no-insomnia groups in single-task (ST) and dual-task (DT) cognitive (number of correct responses) performance. (C) Dual-task cost (DTC), for gait speed and number of correct responses in the cognitive subtraction task. $*_{p}<0.05, * * p<0.01, *^{*} * p<0.00$ I. Error bars show standard error.

an accumulation of nonspecific abnormalities distributed across the central nervous system (CNS), ${ }^{63}$ and it may be challenging to extract data on specific isolated regions.

Physiologically, cardiometabolic risk factors for noncommunicable diseases (NCDs), including high blood pressure, obesity, and glycemic markers, are strongly related to both sleep ${ }^{64,65}$ and gait abnormalities. ${ }^{66}$ The suggested mechanisms underlying these pathologies involve high inflammatory markers. For example, poor sleep is associated with increased serum inflammatory marker levels among obese adults: Poor sleepers had significantly larger IL-6 responses to cognitive stressors compared to good sleepers. ${ }^{67}$ An inverse association was found between sleep quality and production of the proinflammatory cytokine IL- $1 \beta,{ }^{68}$ and sleep loss has been shown to alter molecular processes that drive cellular immune activation and induce inflammatory cytokines. ${ }^{69}$ Inflammation can also alter mobility patterns either by its effect on executive functions ${ }^{70}$ or directly by its effect on gait. The inflammatory marker IL-6 was found to be associated with gait performance in communitydwelling seniors and predicted the risk of gait speed decline in adults aged 70 and older. ${ }^{71}$ In addition, high inflammation was associated with slower walking speed. $^{72}$

Emotional regulation disturbance is another potential mechanism, which has been widely linked separately to both sleep and gait. ${ }^{73}$ Indeed, mood and specifically anxiety and depression are associated with an increased risk of falls as well as of insomnia among older adults. Associations between depression and poor sleep are well documented, ${ }^{74,75}$ and the effects of anxiety and depression on gait have been widely described. ${ }^{76,77}$ Mood problems accompanied by insomnia may serve as the entry point to a vicious cycle that leads to social restriction, deteriorated executive function, ${ }^{78}$ reduced mobility followed by fear of 


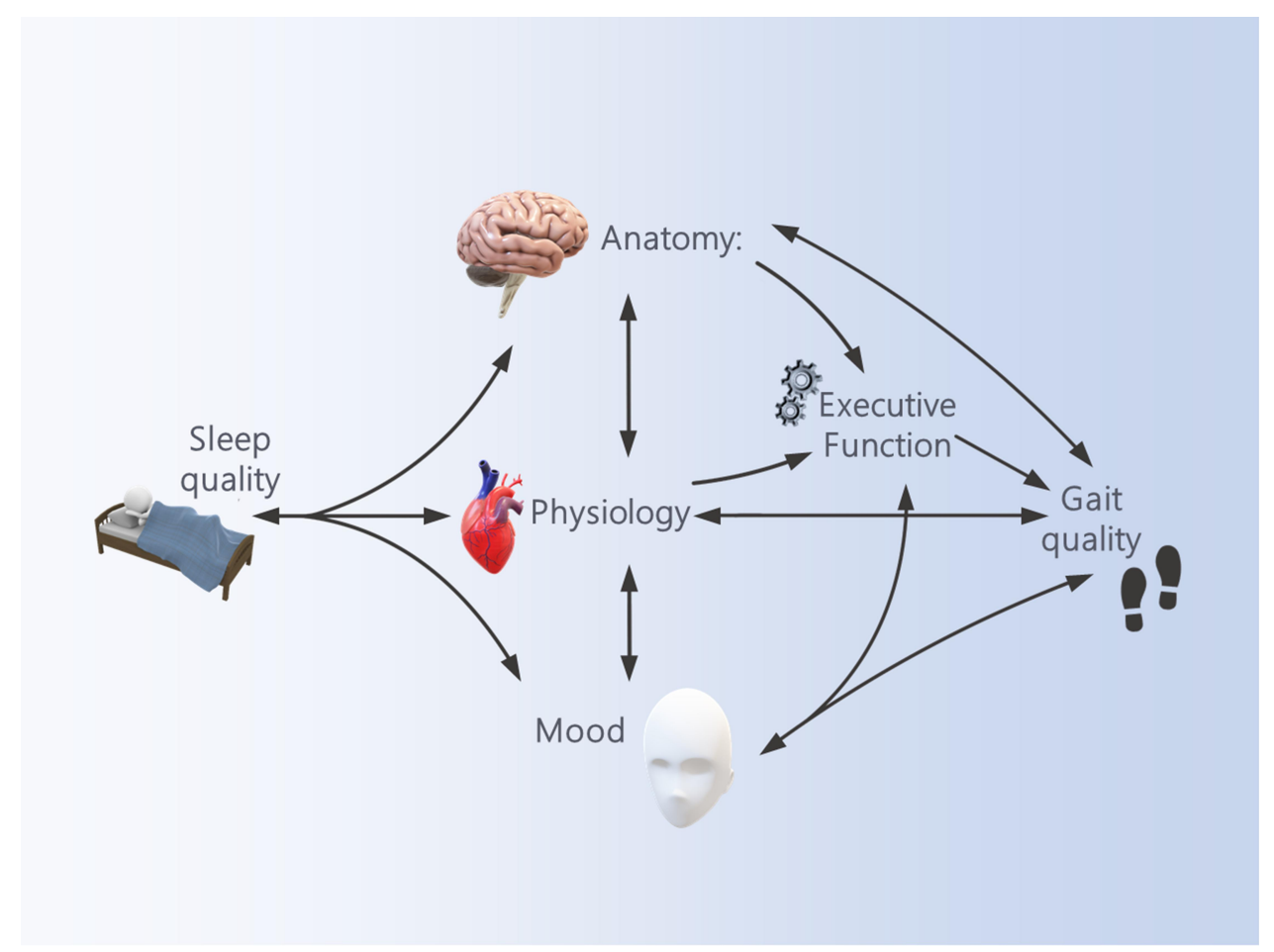

Figure 2 A theoretical model proposing underlying potential mechanisms explaining the link between sleep quality, executive function, and gait quality.

falling, and higher risk of falls. ${ }^{78-80}$ As such, untangling these undesired effects and adverse health outcomes that are associated with sleep and gait abnormalities can significantly improve quality of life in many aspects.

The link between sleep quality and gait performance demonstrated in this study has meaningful clinical implications. Since gait abnormalities and reduced sleep quality are both associated with increased fall risk, sleep disturbances have the potential of being markers for increased fall risk and vice versa. In addition, impairment in gait performance can be used as a potential indicator of overlooked sleep problems. The understanding that there are shared mechanisms underlying the association between insomnia and reduced gait quality can lead to novel approaches for early clinical interventions: despite the well-known link between insomnia and falls in older adults, to the best of our knowledge, no study has tested the effects of improving sleep quality on gait or the effects of improving gait on insomnia. Treatment strategies with demonstrated efficacy for insomnia include pharmacological and non-pharmacological cognitive and behavioural approaches, ${ }^{81}$ and different studies have suggested interventions to improve gait performance ${ }^{82}$ - the efficacy of such sleep interventions on gait and vice versa should be further studied.
Our study has some limitations. The relatively small sample size, and its primary composition of highfunctioning, community-dwelling adults, limits the generalization of our findings. The use of two different actigraphy devices in each study group is an obvious methodological limitation; however, obtained sleep measures were used only descriptively and selection criteria for sleep status (insomnia/no insomnia) in each group were based on both objective measures and self-report. In addition, studies in young individuals comparing these two commonly used actigraphy brands demonstrated high agreement. ${ }^{37,83}$ Data on sleep medication use were not specific; even so, sensitivity analysis excluding all participants who reported sleep medication use did not alter our findings. Furthermore, a recent systematic review of zolpidem, the most commonly prescribed sleep medication for insomnia in older adults, found low risk for residual daytime sleepiness, negative psychomotor effects, or falls when used with the recommended dose. ${ }^{84}$ Nevertheless, future studies should carefully collect information regarding specific medication use and further control the effect of other comorbidities on the relationship between sleep and gait. In addition, due to the small sample size, we could not for potential other confounding variables, eg, comorbid and life-style factors. Longitudinal designs as well as randomized clinical trials 
may collect data on falls and further untangle the nature of these relationships and infer causality and underlying mechanisms between the sleep and gait domains.

In conclusion, we aimed to compare gait and cognitive performance under single and dual-task conditions among community-dwelling older adults with and without insomnia. Our study demonstrates that older adults with insomnia have lower gait speed and poorer cognitive performance during single and dual tasks as well as an unsafe and potentially risky pattern of task prioritization during walking, compared to their counterparts without insomnia. These findings indicate that those with insomnia are at an increased risk of falls, which may offer insight into the underpinnings of the association between insomnia and falls in older adults. We have postulated a theoretical model to describe interconnected anatomical, physiological, and emotional mechanisms underlying this link. Future studies should further confirm the proposed model by focusing on its specific mechanisms among different populations, such as low-functioning older adults and individuals across the lifespan with insomnia and/ or chronic sleep loss. This study may pave the way towards the development of effective interventions that may improve sleep quality and gait performance.

\section{Acknowledgments}

This study is partly supported by the Israeli Ministry of Science and Technology, Grant 3-13607.

\section{Disclosure}

The authors report no conflicts of interest in this work.

\section{References}

1. Altena E, Baglioni C, Espie CA, et al. Dealing with sleep problems during home confinement due to the COVID-19 outbreak: practical recommendations from a task force of the European CBT-I academy. J Sleep Res. 2020;29(4). doi:10.1111/jsr.13052

2. Yu BYM, Yeung W-F, Lam JC-S, et al. Prevalence of sleep disturbances during COVID-19 outbreak in an urban Chinese population: a cross-sectional study. Sleep Med. 2020;74:18-24. doi:10.1016/j. sleep.2020.07.009

3. Ellis JG, Perlis ML, Neale LF, Espie CA, Bastien CH. The natural history of insomnia: focus on prevalence and incidence of acute insomnia. J Psychiatr Res. 2012;46(10):1278-1285. doi:10.1016/j. jpsychires.2012.07.001

4. Morin CM, Drake CL, Harvey AG, et al. Insomnia disorder. Nat Rev Dis Prim. 2015;1. doi:10.1038/nrdp.2015.26

5. Ohayon MM. Epidemiology of insomnia: what we know and what we still need to learn. Sleep Med Rev. 2002;6(2):97-111. doi:10.1053/ smrv.2002.0186

6. Riemann D, Nissen C, Palagini L, Otte A, Perlis ML, Spiegelhalder K. The neurobiology, investigation, and treatment of chronic insomnia. Lancet Neurol. 2015;14(5):547-558. doi:10.1016/S1474-4422(15) 00021-6
7. Fortier-Brochu É, Morin CM. Cognitive impairment in individuals with insomnia: clinical significance and correlates. Sleep. 2014;37 (11):1787-1798. doi:10.5665/sleep.4172

8. Fortier-Brochu É, Beaulieu-Bonneau S, Ivers H, Morin CM. Insomnia and daytime cognitive performance: a meta-analysis. Sleep Med Rev. 2012;16(1):83-94. doi:10.1016/j.smrv.2011.03.008

9. Li MJ, Kechter A, Olmstead RE, Irwin MR, Black DS. Sleep and mood in older adults: coinciding changes in insomnia and depression symptoms. Int Psychogeriatr. 2018;30(3):431-435. doi:10.1017/ S1041610217001454

10. Stone KL, Ancoli-Israel S, Blackwell T. Actigraphy-measured sleep characteristics and risk of falls in older women. Arch Intern Med. 2008;168(16):1768-1775. doi:10.1001/archinte.168.16.1768

11. Takada S, Yamamoto Y, Shimizu S, et al. Association between subjective sleep quality and future risk of falls in older people: results from LOHAS. J Gerontol a Biol Sci. 2018;73(9):1205-1211. doi:10.1093/gerona/glx123

12. Stone KL, Blackwell TL, Ancoli-Israel S. Sleep disturbances and risk of falls in older community-dwelling men: the outcomes of sleep disorders in older men (MrOS sleep) study. $J$ Am Geriatr Soc. 2014;62(2):299-305. doi:10.1111/jgs. 12649

13. Hill EL, Cumming RG, Lewis R, Carrington S, Le Couteur DG. Sleep disturbances and falls in older people. J Gerontol A Biol Sci. 2007;62(1):62-66. doi:10.1093/gerona/62.1.62

14. Helbig AK, Döring A, Heier M. Association between sleep disturbances and falls among the elderly: results from the German cooperative health research in the region of Augsburg-age study. Sleep Med. 2013;14(12):1356-1363. doi:10.1016/j.sleep.2013.09.004

15. Robillard R, Prince F, Filipini D, Carrier J. Aging worsens the effects of sleep deprivation on postural control. Dickson CT ed. PLoS One. 2011;6(12):e28731. doi:10.1371/journal.pone.0028731

16. Aguiar SA, Barela JA. Sleep deprivation affects sensorimotor coupling in postural control of young adults. Neurosci Lett. 2014;574:47-52. doi:10.1016/j.neulet.2014.05.028

17. Stevens D, Jackson B, Carberry J, et al. The impact of obstructive sleep apnea on balance, gait, and falls risk: a narrative review of the literature. J Gerontol A. 2020;75(12):2450-2460. doi:10.1093/gerona/glaa014

18. Gallea C, Ewenczyk C, Degos B, et al. Pedunculopontine network dysfunction in Parkinson's disease with postural control and sleep disorders. Mov Disord. 2017;32(5):693-704. doi:10.1002/mds.26923

19. Montesinos L, Castaldo R, Cappuccio FP, Pecchia L. Day-to-day variations in sleep quality affect standing balance in healthy adults. Sci Rep. 2018;8(1):17504. doi:10.1038/s41598-018-36053-4

20. Agmon M, Shochat T, Kizony R. Sleep quality is associated with walking under dual-task, but not single-task performance. Gait Posture. 2016;49:127-131. doi:10.1016/j.gaitpost.2016.06.016

21. Clark DJ. Automaticity of walking: functional significance, mechanisms, measurement and rehabilitation strategies. Front Hum Neurosci. 2015;9:246. doi:10.3389/fnhum.2015.00246

22. Belghali M, Chastan N, Cignetti F, Davenne D, Decker LM. Loss of gait control assessed by cognitive-motor dual-tasks: pros and cons in detecting people at risk of developing Alzheimer's and Parkinson's diseases. GeroScience. 2017;39(3):305-329. doi:10.1007/s11357017-9977-7

23. Rasmussen LJH, Caspi A, Ambler A, et al. Association of neurocognitive and physical function with gait speed in midlife. JAMA Netw open. 2019;2(10):e1913123. doi:10.1001/jamanetworkopen.2019.13123

24. Springer S, Giladi N, Peretz C, Yogev G, Simon ES, Hausdorff JM. Dual-tasking effects on gait variability: the role of aging, falls, and executive function. Mov Disord. 2006;21(7):950-957. doi:10.1002/ mds. 20848

25. Verghese J, Buschke H, Viola L, et al. Validity of divided attention tasks in predicting falls in older individuals: a preliminary study. $J$ Am Geriatr Soc. 2002;50(9):1572-1576. doi:10.1046/j.15325415.2002.50415.x 
26. Bayot M, Dujardin K, Dissaux L, et al. Can dual-task paradigms predict falls better than single task? - a systematic literature review. Neurophysiol Clin. 2020;50(6):401-440. doi:10.1016/j.neucli.2020.10.008

27. Kelly VE, Janke AA, Shumway-Cook A. Effects of instructed focus and task difficulty on concurrent walking and cognitive task performance in healthy young adults. Exp Brain Res. 2010;207(1-2):65-73. doi:10.1007/s00221-010-2429-6

28. Al-Yahya E, Dawes H, Smith L, Dennis A, Howells K, Cockburn J. Cognitive motor interference while walking: a systematic review and meta-analysis. Neurosci Biobehav Rev. 2011;35(3):715-728. doi:10.1016/j.neubiorev.2010.08.008

29. Malcolm BR, Foxe JJ, Butler JS, De Sanctis P. The aging brain shows less flexible reallocation of cognitive resources during dual-task walking: a mobile brain/body imaging (MoBI) study. Neuroimage. 2015;117:230-242. doi:10.1016/j.neuroimage.2015.05.028

30. Menant JC, Sturnieks DL, Brodie MAD, Smith ST, Lord SR. Visuospatial tasks affect locomotor control more than nonspatial tasks in older people. PLoS One. 2014;9(10):e109802. doi:10.1371/ journal.pone.0109802

31. Corp DT, Youssef GJ, Clark RA. Reduced motor cortex inhibition and a 'cognitive-first' prioritisation strategy for older adults during dual-tasking. Exp Gerontol. 2018;113:95-105. doi:10.1016/j. exger.2018.09.018

32. Buysse DJ, Ancoli-Israel S, Edinger JD, Lichstein KL, Morin CM. Recommendations for a standard research assessment of insomnia Sleep. 2006;29(9):1155-1173. doi:10.1093/sleep/29.9.1155

33. Lichstein KL, Durrence HH, Taylor DJ, Bush AJ, Riedel BW. Quantitative criteria for insomnia. Behav Res Ther. 2003;41 (4):427-445. doi:10.1016/S0005-7967(02)00023-2

34. Buysse DJ, Reynolds CF, Monk TH, Berman SR, Kupfer DJ. The Pittsburgh sleep quality index: a new instrument for psychiatric practice and research. Psychiatry Res. 1989;28(2):193-213. doi:10.1016/0165-1781(89)90047-4

35. Shochat T, Tzischinsky O, Oksenberg A, Peled R. Validation of the Pittsburgh sleep quality index Hebrew translation (PSQI-H) in a sleep clinic sample. Isr Med Assoc J. 2007;9(12):853-856.

36. Sadeh A, Acebo C. The role of actigraphy in sleep medicine. Sleep Med Rev. 2002;6(2):113-124. doi:10.1053/smrv.2001.0182

37. Weiss AR, Johnson NL, Berger NA, Redline S. Validity of activity-based devices to estimate sleep. J Clin Sleep Med. 2010;6 (4):336-342. doi: $10.5664 /$ jcsm. 27874

38. Mantua J, Gravel N, Spencer RMC. Reliability of sleep measures from four personal health monitoring devices compared to research-based actigraphy and polysomnography. Sensors. 2016;16 (5):646. doi:10.3390/s16050646

39. Plummer P, Eskes G. Measuring treatment effects on dual-task performance: a framework for research and clinical practice. Front Hum Neurosci. 2015;9(APR). doi:10.3389/fnhum.2015.00225

40. Yogev-Seligmann G, Hausdorff JM, Giladi N. Do we always prioritize balance when walking? Towards an integrated model of task prioritization. Mov Disord. 2012;27(6):765-770. doi:10.1002/ mds. 24963

41. Liu X, Sun B, Zhang Z, Wang Y, Tang H, Zhu T. Gait can reveal sleep quality with machine learning models. Ostadabbas $\mathrm{S}$ ed. PLoS One. 2019;14(9):e0223012. doi:10.1371/journal.pone.0223012

42. O’Dowd S, Galna B, Morris R, et al. Poor sleep quality and progression of gait impairment in an incident Parkinson's disease cohort. J Parkinsons Dis. 2017;7(3):465-470. doi:10.3233/JPD-161062

43. Bernstein JPK, De Vito A, Weitzner DS, et al. Examining relationships between multiple self-reported sleep measures and gait domains in cognitively healthy older adults. Gerontology. 2020;66(1):47-54. doi:10.1159/000499737

44. Bernstein JPK, Calamia M, Keller JN. Multiple self-reported sleep measures are differentially associated with cognitive performance in community-dwelling nondemented elderly. Neuropsychology. 2018;32(2):220-229. doi:10.1037/neu0000407
45. Mcdade EM, Boot BP, Christianson TJH, et al. Subtle gait changes in patients with REM sleep behavior disorder. Mov Disord. 2013;28 (13):1847-1853. doi:10.1002/mds.25653

46. Allali G, Perrig S, Cleusix M, et al. Gait abnormalities in obstructive sleep apnea and impact of continuous positive airway pressure. Respir Physiol Neurobiol. 2014;201:31-33. doi:10.1016/j. resp.2014.06.012

47. Goldman SE, Stone KL, Ancoli-Israel S. Poor sleep is associated with poorer physical performance and greater functional limitations in older women. Sleep. 2007;30(10):1317-1324. doi:10.1093/sleep/ 30.10 .1317

48. Nakakubo S, Doi T, Shimada H, et al. The association between excessive daytime sleepiness and gait parameters in community-dwelling older adults: cross-sectional findings from the Obu study of health promotion for the elderly. J Aging Health. 2018;30(2):213-228. doi:10.1177/0898264316673253

49. Ble A, Volpato S, Zuliani G, et al. Executive function correlates with walking speed in older persons: the InCHIANTI study. J Am Geriatr Soc. 2005;53(3):410-415. doi:10.1111/j.1532-5415.2005.53157.x

50. Yogev G, Plotnik M, Peretz C, Giladi N, Hausdorff JM. Gait asymmetry in patients with Parkinson's disease and elderly fallers: when does the bilateral coordination of gait require attention? Exp Brain Res. 2007;177(3):336-346. doi:10.1007/s00221-006-0676-3

51. Yogev-Seligmann G, Hausdorff JM, Giladi N. The role of executive function and attention in gait. Mov Disord. 2008;23(3):329-342. doi: $10.1002 / \mathrm{mds} .21720$

52. Zimmerman ME, Bigal ME, Katz MJ, Brickman AM, Lipton RB. Sleep onset/maintenance difficulties and cognitive function in nondemented older adults: the role of cognitive reserve. $J$ Int Neuropsychol Soc. 2012;18(3):461-470. doi:10.1017/ S1355617711001901

53. Haimov I, Shatil E, Laks J. Cognitive training improves sleep quality and cognitive function among older adults with insomnia. PLoS One. 2013;8(4):e61390. doi:10.1371/journal.pone.0061390

54. Contreras-González N, Téllez-Alanís B, Haro R, Jiménez-Correa U, Poblano A. Executive dysfunction in patients with chronic primary insomnia treated with clonazepam. Neurol Res. 2015;37 (12):1047-1053. doi:10.1080/01616412.2015.1114740

55. Wei Y, Leerssen J, Wassing R, Stoffers D, Perrier J, Van Someren EJW. Reduced dynamic functional connectivity between salience and executive brain networks in insomnia disorder. $J$ Sleep Res. 2020;29(2). doi:10.1111/jsr.12953

56. Haimov I, Hanuka E, Horowitz Y. Chronic insomnia and cognitive functioning among older adults. Behav Sleep Med. 2008;6(1):32-54. doi:10.1080/15402000701796080

57. Holtzer R, Wang C, Lipton R, Verghese J. The protective effects of executive functions and episodic memory on gait speed decline in aging defined in the context of cognitive reserve. J Am Geriatr Soc. 2012;60(11):2093-2098. doi:10.1111/j.15325415.2012.04193.x

58. Lewis SJG. Neurological update: emerging issues in gait disorders. $J$ Neurol. 2015;262(6):1590-1595. doi:10.1007/s00415-015-7686-7

59. Elcombe EL, Lagopoulos J, Duffy SL, et al. Hippocampal volume in older adults at risk of cognitive decline: the role of sleep, vascular risk, and depression. J Alzheimers Dis. 2015;44(4):1279-1290. doi:10.3233/JAD-142016

60. O'Shea A, Cohen RA, Porges EC, Nissim NR, Woods AJ. Cognitive aging and the hippocampus in older adults. Front Aging Neurosci. 2016;8. doi:10.3389/fnagi.2016.00298.

61. Allali G, Van Der Meulen M, Beauchet O, Rieger SW, Vuilleumier P, Assal F. The neural basis of age-related changes in motor imagery of gait: an fMRI study. J Gerontol A Biol Sci. 2014;69(11):1389-1398. doi:10.1093/gerona/glt207

62. Rosso AL, Verghese J, Metti AL, et al. Slowing gait and risk for cognitive impairment. Neurology. 2017;89(4):336-342. doi:10.1212/ WNL.0000000000004153 
63. Rosso AL, Studenski SA, Chen WG, et al. Aging, the central nervous system, and mobility. J Gerontol A Biol Sci Med Sci. 2013;68 (11):1379-1386. doi:10.1093/gerona/glt089

64. Fernandez-Mendoza J, Vgontzas AN, Liao D, et al. Insomnia with objective short sleep duration and incident hypertension: the penn state cohort. Hypertension. 2012;60(4):929-935. doi:10.1161/ HYPERTENSIONAHA.112.193268

65. Silva-Costa A, Rotenberg L, Nobre AA, et al. Sex differences in the association between self-reported sleep duration, insomnia symptoms and cardiometabolic risk factors: cross-sectional findings from Brazilian longitudinal study of adult health. Arch Public Health. 2020;78(1). doi:10.1186/s13690-020-00429-8

66. Veronese N, Stubbs B, Volpato S, et al. Association between gait speed with mortality, cardiovascular disease and cancer: a systematic review and meta-analysis of prospective cohort studies. $\mathrm{J} \mathrm{Am} \mathrm{Med}$ Dir Assoc. 2018;19(11):981-988.e7. doi:10.1016/j. jamda.2018.06.007

67. Huang Y, Jiang Y, Zhu M. The relationship between global sleep score and inflammatory markers in obese adults from the United States. Nat Sci Sleep. 2019;11:317-324. doi:10.2147/NSS.S220436

68. Prather AA, Marsland AL, Hall M, Neumann SA, Muldoon MF, Manuck SB. Normative variation in self-reported sleep quality and sleep debt is associated with stimulated pro-inflammatory cytokine production. Biol Psychol. 2009;82(1):12-17. doi:10.1016/j. biopsycho.2009.04.008

69. Irwin MR, Wang M, Campomayor CO, Collado-Hidalgo A, Cole S. Sleep deprivation and activation of morning levels of cellular and genomic markers of inflammation. Arch Intern Med. 2006;166 (16):1756-1762. doi:10.1001/archinte.166.16.1756

70. Schram MT, Euser SM, De Craen AJM, et al. Systemic markers of inflammation and cognitive decline in old age. J Am Geriatr Soc. 2007;55(5):708-716. doi:10.1111/j.1532-5415.2007.01159.x

71. Verghese J, Holtzer R, Oh-Park M, Derby CA, Lipton RB, Wang C. Inflammatory markers and gait speed decline in older adults. J Gerontol A Biol Sci. 2011;66 A(10):1083-1089. doi:10.1093/gerona/glr099

72. Lasselin J, Sundelin T, Wayne PM, et al. Biological motion during inflammation in humans. Brain Behav Immun. 2020;84:147-153. doi:10.1016/j.bbi.2019.11.019

73. Dantzer R, O’Connor JC, Freund GG, Johnson RW, Kelley KW. From inflammation to sickness and depression: when the immune system subjugates the brain. Nat Rev Neurosci. 2008;9(1):46-56. doi:10.1038/nrn2297
74. Furihata R, Hall MH, Stone KL, et al. Aggregate measure of sleep health. Sleep. 2017;40(3). doi:10.1093/sleep/zsw075

75. Paudel ML, Taylor BC, Diem SJ, et al. Association between depressive symptoms and sleep disturbances in community-dwelling older men. J Am Geriatr Soc. 2008;56(7):1228-1235. doi:10.1111/j.15325415.2008.01753.x

76. Atkinson HH, Rosano C, Simonsick EM, et al. Cognitive function, gait speed decline, and comorbidities: the health, aging and body composition study. J Gerontol A Biol Sci. 2007;62(8):844-850. doi:10.1093/gerona/62.8.844

77. Hausdorff JM, Schweiger A, Herman T, Yogev-Seligmann G, Giladi N. Dual-task decrements in gait: contributing factors among healthy older adults. J Gerontol A Biol Sci. 2008;63(12):1335-1343. doi:10.1093/gerona/63.12.1335

78. Best JR, Davis JC, Liu-Ambrose T. Longitudinal analysis of physical performance, functional status, physical activity, and mood in relation to executive function in older adults who fall. $J$ Am Geriatr Soc. 2015;63(6):1112-1120. doi:10.1111/jgs.13444

79. Hajek A, Bock JO, König HH. Psychological correlates of fear of falling: findings from the German aging survey. Geriatr Gerontol Int. 2018;18(3):396-406. doi:10.1111/ggi.13190

80. Holloway KL, Williams LJ, Brennan-Olsen SL, et al. Anxiety disorders and falls among older adults. J Affect Disord. 2016;205:20-27. doi:10.1016/j.jad.2016.06.052

81. Boland E, Goldschmied J, Kayser MS, Gehrman PR. Precision medicine for insomnia. Sleep Med Clin. 2019;14(3):291-299. doi:10.1016/j.jsmc.2019.04.001

82. Yogev-Seligmann G, Giladi N, Brozgol M, Hausdorff JM. A training program to improve gait while dual tasking in patients with Parkinson's disease: a pilot study. Arch Phys Med Rehabil. 2012;93 (1):176-181. doi:10.1016/j.apmr.2011.06.005

83. Tonetti L, Pasquini F, Fabbri M, Belluzzi M, Natale V. Comparison of two different actigraphs with polysomnography in healthy young subjects. Chronobiol Int. 2008;25(1):145-153. doi:10.1080/ 07420520801897228

84. Machado FV, Louzada LL, Cross NE, Camargos EF, Dang-Vu TT, Nóbrega OT. More than a quarter century of the most prescribed sleeping pill: systematic review of zolpidem use by older adults. Exp Gerontol. 2020;136:110962. doi:10.1016/j.exger.2020.110962
Nature and Science of Sleep

\section{Publish your work in this journal}

Nature and Science of Sleep is an international, peer-reviewed, open access journal covering all aspects of sleep science and sleep medicine, including the neurophysiology and functions of sleep, the genetics of sleep, sleep and society, biological rhythms, dreaming, sleep disorders and therapy, and strategies to optimize healthy sleep.
The manuscript management system is completely online and includes a very quick and fair peer-review system, which is all easy to use. Visit http://www.dovepress.com/testimonials.php to read real quotes from published authors. 